\title{
Regulation of arginine metabolism by dietary fatty acids: involvement of PPAR $\alpha$
}

\author{
N. Guelzim ${ }^{1}$, F. Mariotti ${ }^{1}$, F. Lasserre ${ }^{2}$, V. Mathé ${ }^{1}$, D. Azzout ${ }^{1}$, T. Pineau ${ }^{2}$, J. F. Huneau $^{1}$ \\ and D. Hermier ${ }^{1}$ \\ ${ }^{1}$ UMR914, Physiology of Nutrition and Ingestive Behaviour, INRA, AgroParisTech, Paris, France \\ and ${ }^{2}$ UR66, Pharmacology-Toxicology, INRA, Toulouse, France
}

\begin{abstract}
As a result of pleiotropic effects, PPAR $\alpha$ ligands, and particularly PUFA, are likely to regulate amino acid metabolism. Arginine (Arg) is of special interest, since it is the precursor of NO, a key player in vascular homeostasis. NO production depends on differential Arg channelling towards either urea via arginase or NO via NO synthase (NOS). These metabolic pathways were investigated in wild-type (WT) and PPAR $\alpha$-null (KO) male mice. They were fed for 8 weeks on lipid-poor diets $(4.8 \%$, w/w) containing either SFA (SFA diet; $93 \%$ fatty acids as SFA) or $18: 3 n-3$ (ALA diet; $60 \%$ fatty acids as $18: 3 n-3$ ). Urinary excretion of urea and nitrates (the final product of NO metabolism) was determined in urine, and urea and amino acid concentrations were assayed in plasma. In addition, the whole-body differential channelling of Arg was assessed by determining the conversion of an intraperitoneal bolus dose of $\left[{ }^{15} \mathrm{~N}_{2}\right.$-guanido]arginine into urinary ${ }^{15} \mathrm{NO}_{3} v$. $\left[{ }^{15} \mathrm{~N}_{2}\right.$ ] urea. Levels of hepatic arginase II protein were assayed by Western blotting. Hepatic levels of arginase II mRNA and endothelial (eNOS) and inducible (iNOS) NOS mRNA were determined by real-time PCR. Differences were significant at $P<0.05$.

For mice fed the ALA diet $(n 6)$ hepatic levels of arginase II protein and mRNA and NOS mRNA were 2-fold lower than those for mice fed the SFA diet. However, plasma concentrations of urea and Arg, urinary concentrations of urea and nitrates, and whole-body conversion of Arg into urea $v$. nitrates did not differ with the diet.

For KO mice arginase II protein and mRNA levels were 2-fold lower than those for the WT groups, with no significant effect on plasma and urine metabolic markers. PPAR $\alpha$ deficiency did not alter eNOS and iNOS expression. In addition, the absence of PPAR $\alpha$ partly abolished the dietary effects, which were less pronounced than those for WT mice. However, because of an interaction between genotype and diet the whole-body conversion of Arg into NO ( $v$. urea) tended to be lower (by $50 \%$ ) in KO mice fed the ALA diet than in those fed the SFA diet.

The regulation of Arg metabolic pathways may be mediated by the nature of the dietary fatty acids. Low dietary levels of $18: 3 n-3$ $(2.9 \%(\mathrm{w} / \mathrm{w}))$ down regulated the key enzymes of both urea and NO synthesis. PPAR $\alpha$ is involved in this regulation, since KO mice were less sensitive to dietary fatty acids. However, metabolic markers of Arg metabolism must be investigated in more relevant models.
\end{abstract}

